\title{
Postura, desenvolvimento e eclosão dos ovos de Dolops carvalhoi Lemos de Castro (Crustácea, Branchiura) em laboratório, parasita de peixes da Amazônia Central
}

\author{
Ana Lúcia Silva Gomes ${ }^{1}$ \\ José Celso de Oliveira Malta ${ }^{1}$
}

\begin{abstract}
Laying, development and hatching of eggs of the Dolops carvalhoi Lemos de Castro (Crustacea, Branchiura), reared in the laboratory, parasite from fishes of Central Amazon. Dolops carvalhoi Lemos de Castro, 1949 was found infesting fish in a fish culture station located in highway AM-010 Manaus-Itacoatiara in the State of Amazonas. Egg laying of D. carvalhoi was studied. Hatching started after 16 days in a temperature span of 26 to $28^{\circ} \mathrm{C}$. Eggs were laid in clusters on hard substrata and covered by a gelatinous material. The number of eggs was 16 to 392 eggs by laying. In the postembryonic development the larvae were hatched from the egg as juveniles (epimorphic development), very similar to the adult shape.

KEY WORDS. Argulidae, Dolops carvalhoi, laying, eggs, fish parasites, Amazon
\end{abstract}

As espécies do gênero Dolops Audouin, 1837 são as únicas da subclasse Branchiura que apresentam maxílulas robustas, dilatadas na base e na extremidade distal com um forte gancho quitinoso, durante toda a vida. $\mathrm{O}$ aparelho bucal não apresenta o estilete anterior ao cone oral. As antenas não possuem o espinho anterior (RINGUELET 1943; LEMOS DE CASTRO 1986). Elas parasitam somente peixes de água doce e a maioria ocorre na América do Sul. Exceto D. ranarum (Stuhlmann, 1891) que parasita peixes da África (MALTA 1982) e D. tasmanianus Fryer, 1968 que é endêmico a Tasmânia (FrYER 1968).

O tipo de postura dos ovos nos branquiúros é único entre os crustáceos. As fêmeas grávidas abandonam o peixe hospedeiro e depositam os ovos sobre substratos sólidos: troncos, pedras e raízes (FRYER 1968; MALTA \& VARELLA 1983; SHAFIR \& VAN As 1986). Estudos com a postura, desenvolvimento e eclosão de ovos entre os branquiúros foram feitos com A. foliaceus (Linnaeus, 1758) por Claus (1875) apud WILSON (1902); A. megalops Wilson, 1902, A. catastomi Dana \& Herrick, 1837 e A. stizostehii Kellicott, 1880 por WILSON (1902); A. americanus Wilson (1904) e A. violaceus Thomsen, 1925 por RINGUELET (1943); A. africanus Thiele, 1900 por FRYER (1956); A. puthenveliensis Ramakrisna, 1959 por THOMAS (1961); A. japonicus Thiele, 1900 por THOMAS (1961) e SHAFIR \& VAN As (1986); A. coregoni Thorell, 1864 por SHIMURA (1981). Somente é conhecido a postura, o desenvolvimento embrionário e o ovo de uma única espécie do gênero Dolops, $D$. ranarum parasita de peixes da África (FRYER 1959; AVENANT et al. 1989).

1) Laboratório de Parasitologia e Patologia de Peixes, Coordenação de Pesquisas em Biologia Aquática, Instituto Nacional de Pesquisas da Amazônia. Caixa Postal 478, 69011-970 Manaus, Amazonas, Brasil. E-mail: analu@interlins.com.br; jcmalta@inpa.gov.br 
A espécie estudada, D. carvalhoi Lemos de Castro 1949, descrita por com base em seis exemplares coletados na confluência dos rios Kuluene com o Xingu, no Estado do Mato Grosso, Brasil. Parasitavam as brânquias de Rhaphiodon vulpinus (Agassiz, 1829) (LEMOS DE CASTRO 1949). Na Amazônia foi coletado no lago Janauacá, um lago de várzea da Amazônia Central localizado na margem direita do rio Solimões/Amazonas no Estado do Amazonas. Apresentou uma sazonalidade na ocorrência, com os maiores índices de infestação na estação da cheia e os menores na seca. D. carvalhoi apresentou uma baixa especificidade parasitária, foi encontrado parasitando sete espécies de peixes (MALTA 1984; MALTA \& VARELLA 1983). Também foi registrada a ocorrência $D$. carvalhoi na Venezuela, embora tenha sido equivocadamente identificado como D. geayi (WEIBEZAHN \& COBO 1964).

\section{MATERIAL E MÉTODOS}

Foram realizadas três coletas em uma estação de piscicultura localizada na estrada AM 010, Manaus-Itacoatiara, km 251. A primeira ocorreu em abril de 1998, quando juvenis de tambaqui, Colossoma macropomum (Cuvier, 1818) foram trazidos para o laboratório para serem utilizados como hospedeiros de D. carvalhoi. Os peixes tinham 50 dias de idade, mediam cinco centímetros de comprimento padrão e seis gramas de peso. Foram transportados vivos, em sacos plásticos de 50 litros, com água aerada. Os sacos foram acondicionados em uma caixa de isopor, com uma fina camada de gelo no fundo.

No laboratório, os peixes foram colocados em caixas de amianto de 1000 litros com filtro biológico e aeração. A água utilizada era proveniente do poço artesiano do Instituto Nacional de Pesquisas da Amazônia (INPA). Os peixes foram alimentados uma vez ao dia, com ração peletizada contendo $30 \%$ de proteína.

Na segunda coleta, em setembro de 1998, quando foram coletados adultos de D. carvalhoi que parasitavam o curimatã, Prochilodus nigricans Agassiz, 1829 e a carpa comum, Cyprinus carpio Linnaeus, 1756. Foram coletados cerca de 90 dolopsídeos. Cada Branchiura foi apreendido com uma pinça fina e colocado em um recipiente plástico de $300 \mathrm{ml}$.

Os 90 exemplares de D. carvalhoi foram transportados para o laboratório, em três sacos plásticos de 20 litros com água e oxigênio, cada um com 30 indivíduos. Para servirem de hospedeiros dos crustáceos durante a viagem, foram colocados dois exemplares de Ctenobrycon spilurus (Valenciennes, 1850) adultos, medindo cerca de $4 \mathrm{~cm}$ de comprimento total, no primeiro saco. No segundo, dois juvenis de tambaqui, com cerca de $20 \mathrm{~cm}$ e no terceiro, nenhum hospedeiro, somente $D$. carvalhoi. Ao chegar ao laboratório, distante $197 \mathrm{~km}$ da estação de piscicultura e após três horas de viagem, os peixes haviam predado todos os exemplares de $D$. carvalhoi. Para constatar a predação, os hospedeiros foram necropsiados e os branquiúros foram encontrados nos estômagos.

Na terceira coleta, em outubro 1998, foram capturados mais exemplares de D. carvalhoi. Foram efetuados os mesmos procedimentos da coleta anterior, exceto que, no transporte para o laboratório, os exemplares de $D$. carvalhoi foram acondicionados em recipientes plásticos de $300 \mathrm{ml}$. 
Para a observação das posturas foram utilizados quatro aquários de 20 litros, providos de filtro biológico e sistema de aeração. Cada recipiente continha três peixes com cerca de 15 parasitas, cada um. Os aquários foram observados diariamente à procura de posturas, que após serem localizadas, eram transferidas para placas de Petri, devidamente identificadas (data, hora, e número de ovos depositados), para posterior acompanhamento do desenvolvimento. As placas de Petri tinham sua água trocada diariamente, evitando o desenvolvimento de colônias de fungos e bactérias. As mensurações da temperatura da água foram feitas três vezes ao dia (08:00, 14:00 e 17:00 horas).

A análise dos dados consistiu de estatística descritiva, número mínimo, máximo e a porcentagem de ovos eclodidos e inviáveis por postura.

\section{RESULTADOS}

Os adultos de Dolops carvalhoi foram coletados parasitando o curimatã, Prochilodus nigricans e a carpa comum, Cyprinus carpio. Estes peixes eram mantidos em um mesmo tanque escavado, na mesma estação de piscicultura onde foram obtidos os tambaquis para o trabalho. A intensidade de infestação variou de 1-60 D. carvalhoi por hospedeiro. Os branquiúros encontravam-se fixados na superfície externa do corpo, principalmente sobre o abdômen e região cefálica. Os jovens foram coletados próximos as nadadeiras peitorais e ventrais.

Durante o transporte, na segunda coleta. Os peixes, Colossoma macropomum e Ctenobrycon spilurus, que foram colocados nos sacos plásticos, com água e oxigênio, para servirem de hospedeiros, predaram todos os exemplares de $D$. carvalhoi. Os exemplares de $C$. spilurus e $C$. macropomum foram necropsiados e os crustáceos foram encontrados nos seus estômagos.

Durante o desenvolvimento do estudo em laboratório, várias vezes foi observado, juvenis de tambaqui predando adultos de $D$. carvalhoi, que nadavam livremente na coluna d'água dos aquários.

Foram utilizadas 25 fêmeas de D. carvalhoi para a obtenção das posturas. Dezoito posturas foram observadas. O número total de ovos foi de 1824 eclodiram 386 e 354 foram inviáveis (Tab. I). A temperatura da água, durante o período do trabalho, variou entre 26 a $28^{\circ} \mathrm{C}$.

A maioria das posturas de $D$. carvalhoi era sempre depositada nas paredes verticais dos aquários e nos tubos de plástico (PVC). Houve sempre uma preferência por superfícies lisas e verticais. Durante o transporte, muitas vezes, as fêmeas grávidas de $D$. carvalhoi faziam suas posturas nas paredes dos recipientes no qual estavam acondicionadas.

As fêmeas de $D$. carvalhoi iniciavam o processo de oviposição, movendo-se para frente no substrato, colocando um ovo de cada vez e formando fileiras. Sem interromper o processo e, a certa distância dessa primeira fita, depositavam uma segunda e assim sucessivamente, compondo uma série de várias fitas de ovos paralelas em forma de cachos. Os ovos eram cobertos por uma camada gelatinosa transparente, que endurecia rapidamente ao entrar em contato com a água, protegendo-os e os fixando firmemente ao substrato escolhido. 
Tabela I. Número total de ovos, porcentagem de inviáveis e eclodidos por postura de Dolops carvalhoi. Os valores entre parênteses representam os números mínimo e máximo de ovos em cada postura observada.

\begin{tabular}{cccc}
\hline Posturas & Total de ovos & Porcentagem de ovos eclodidos & Porcentagem de ovos inviáveis \\
\hline 1 & 96 & 12,5 & 87,5 \\
2 & 32 & 40,6 & 59,4 \\
3 & 109 & 0,0 & 100,0 \\
4 & 114 & 0,0 & 100,0 \\
5 & 101 & 7,9 & 92,1 \\
6 & 61 & 24,6 & 75,4 \\
7 & 38 & 2,6 & 97,4 \\
8 & $(16)$ & 12,5 & 87,5 \\
9 & 57 & 35,1 & 64,9 \\
10 & $(392)$ & 1,3 & 98,7 \\
11 & 120 & 34,2 & 65,8 \\
12 & 255 & 40,0 & 60,0 \\
13 & 110 & 60,9 & 39,1 \\
14 & 73 & 12,3 & 87,7 \\
15 & 58 & 12,1 & 87,9 \\
16 & 84 & 0,0 & 100,0 \\
17 & 79 & 51,9 & 48,1 \\
18 & 29 & 37,9 & 62,1 \\
\hline Total & 1824 & - & - \\
\hline
\end{tabular}

Os ovos de D. carvalhoi eram elipsóides. Imediatamente após a postura a coloração dos ovos era amarela-esbranquiçada. Posteriormente iam escurecendo, ficando amarelo escuro e finalmente castanho. Os ovos que cessavam seu desenvolvimento, tornavam-se inviáveis, apresentavam uma coloração negra.

O número de ovos, por postura de $D$. carvalhoi, variou de 16-392. Não foi possível separar quais posturas eram de fêmeas adultas grandes das fêmeas adultas pequenas. Mas, pela variação numérica pode-se deduzir que os valores próximos ao mínimo seriam das mais fêmeas adultas pequenas e nos próximos ao máximo das fêmeas adultas grandes.

Durante o desenvolvimento embrionário de $D$. carvalhoi, a primeira estrutura que pôde ser identificada macroscopicamente por transparência, no interior do ovo, foram os olhos do embrião, observados somente a partir do nono dia da postura (Fig. 1).

O desenvolvimento embrionário completo de $D$. carvalhoi foi de 16 dias a uma temperatura de $28^{\circ} \mathrm{C}$. O tipo de desenvolvimento foi epimórfico.

\section{DISCUSSÃO}

A especificidade parasitária de $D$. carvalhoi no ambiente natural é baixa, foi encontrado parasitando sete espécies de peixes de quatro famílias (MALTA \& VARELLA 1983). Neste trabalho, D. carvalhoi parasitou dois novos hospedeiros: o curimatã, Prochilodus nigricans e a carpa Cyprinus carpio. Este é o primeiro registro de uma espécie de crustáceo parasita, da fauna brasileira, parasitando uma espécie de peixe exótica ( $C$. carpio) e também o primeiro registro de $D$. carvalhoi parasitando peixes cultivados. 


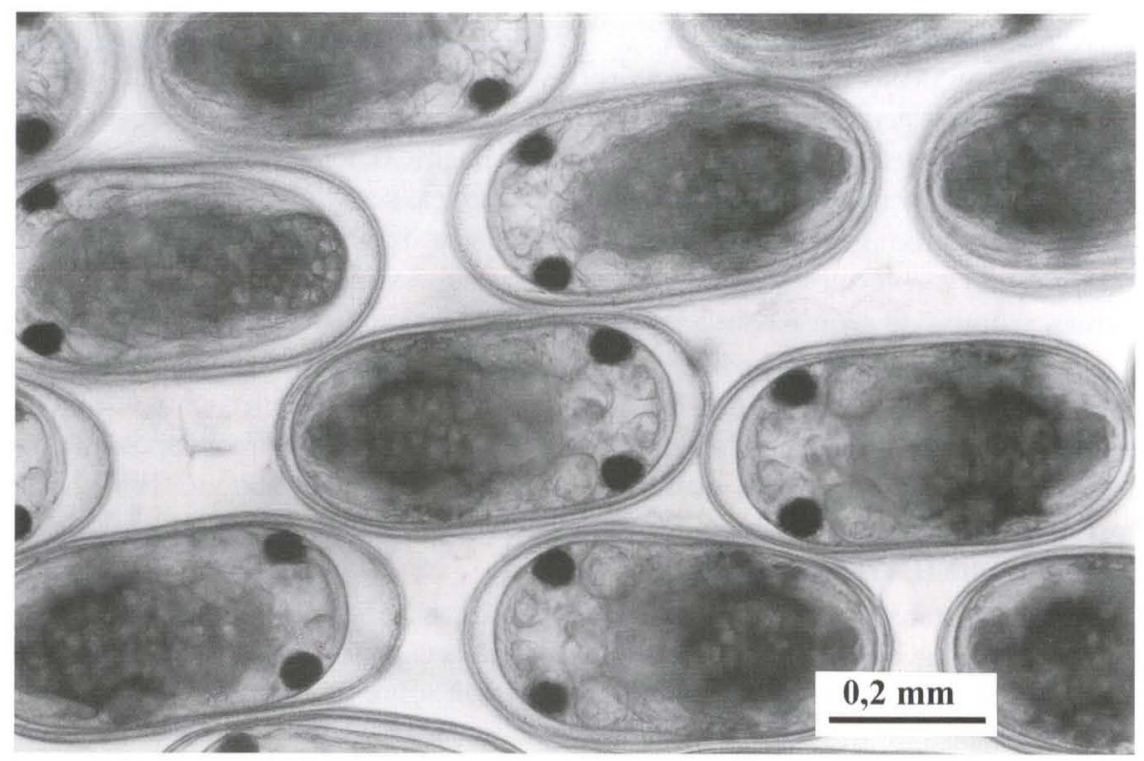

Fig. 1. Postura de Dolops carvalhoi evidenciando o formato, a coloração dos ovos, o posicionamento e os olhos do embrião.

A predação de Branchiura por peixes foi observado em peixes ornamentais da família Cichlidae. Exemplares menores de Pterophyllum scalare Lichtestein, 1801, retiravam Argulus sp. dos peixes maiores. Não houve evidência, de que os argulídeos foram ingeridos e se a alimentação foi a primeira intenção do peixe (KABATA 1970).

Neste trabalho foi verificado a predação de $D$. carvalhoi por Colossoma macropomum e Ctenobrycon spilurus, durante o transporte dos dolopsídeos da estação de piscicultura para o laboratório. Também, durante os trabalhos no laboratório, várias vezes foram observados juvenis de tambaqui predando adultos de $D$. carvalhoi que nadavam livremente na coluna d'água dos aquários. Trata-se do primeiro registro de peixes alimentando-se de Branchiura fora do hospedeiro.

Os branquiúros são únicos na maneira de fazerem suas posturas. As fêmeas abandonam seus hospedeiros e depositam os ovos sobre pedras e troncos (WILSON 1902; RINGUELET 1943). Na postura de A. violaceus os ovos eram postos um a um, em uma ou mais fileiras, cobertos por uma camada gelatinosa que endurecia imediatamente, formando uma casca resistente que os colavam fortemente ao substrato (RINGUELET 1943).

Na postura de A. japonicus os ovos eram colocados em uma única fileira, em forma de fita, as vezes em fileiras múltiplas (ThOMAS 1961). Uma fêmea, independente do tamanho, punha entre 9-11 fitas contendo 5-226 ovos cada, apresentavam uma clivagem longitudinal e eram cobertos por uma camada de material gelatinoso, que prendia fortemente os ovos ao substrato (SHAFIR \& VAN As 1986). 
Nas oviposições de A. megalops os ovos eram dispostos em várias fileiras em linha reta (WILSON 1902). Para A. africanus os ovos eram depositados em uma fileira, podendo ocorrer outras ao lado da primeira (FRYER 1956). Nas de $A$. violaceus os ovos também eram postos em fita única, se a fêmea não encontrasse nenhum obstáculo durante a postura. Na existência de obstáculo, o processo era interrompido e a postura se iniciava mais adiante. Assim, as fitas apresentaram formas distintas de acordo com a topografia da superfície (RINGUELET 1943).

Na postura de D. ranarum os ovos eram depositados em fileiras, formando, no final do processo, uma série de fitas paralelas que resultavam em cachos. Os ovos eram cobertos por uma camada gelatinosa transparente que endurecia após quatro horas, fixando firmemente a postura no substrato (AVENANT et al. 1989). As posturas de $D$. ranarum eram em forma de cachos e a camada gelatinosa endurecia após uma hora (FRYER 1959).

Neste trabalho com $D$. carvalhoi as posturas foram muito semelhantes as de D. ranarum, inclusive a disposição das fileiras em forma de cachos. Já o processo de endurecimento da camada gelatinosa sobre os ovos, foi semelhante ao encontrado por RINGUELET (1943) em A. violaceus e WILSON (1902) em A. catastomi. A camada gelatinosa em contato com a água, formava uma densa cobertura, imediatamente após a postura e não demorava de uma ou quatro horas para endurecer, como em $D$. ranarum.

O local preferido para as posturas de A. violaceus foram as paredes verticais dos aquários (RINGUELET 1943). O mesmo encontrou FrYer (1956) com A. africanus. Já nas posturas de $D$. ranarum os ovos eram sempre depositados sobre pedras e, em sua ausência, nas pedras artificiais usadas para aeração, jamais nas paredes de vidro do aquário (AvENANT et al. 1989). Neste trabalho com D. carvalhoi os resultados foram semelhantes aos de Ringuelet, a maioria das posturas era depositada nas paredes verticais dos aquários.

Fêmeas grávidas de $A$. violaceus capturadas e colocadas em recipientes para transporte, imediatamente iniciavam o processo de postura sobre as paredes de vidro (RINGUELET 1943). A. africanus apresentou o mesmo comportamento (FRYER 1956). O mesmo foi observado em D. ranarum (FRYER 1956; AvENANT et al. 1989), exceto que, os ovos eram depositados nas tampas não translúcidas dos recipientes (AVENANT et al. 1989). D. carvalhoi apresentou o mesmo comportamento de $A$. violaceus e A. africanus.

O número de ovos depositados varia de espécie para espécie e, dentro da mesma espécie, varia entre fêmeas adultas grandes e fêmeas adultas pequenas. Em A. violaceus o número de ovos variou de 50-70 nas fêmeas adultas grandes e de 35-40 nas fêmeas adultas pequenas (RINGUELET 1943). Em A. megalops foi de 60 ovos (WILSON 1902). Em A. puthenveliensis Ramakrisna, 1959 de 140-204, nas fêmeas adultas grandes e de 20-25 nas fêmeas adultas pequenas (THOMAS 1961). O número total de ovos por postura para A. japonicus variou de 12-226 (SHAFIR \& VAN As 1986). O de A. foliaceus foi de 100 (Claus 1875 apud WILSON 1902), de 400 (JURINE 1806 apud WILSON 1902) e de 15-200 (BOWER-SHORE 1940 apud AvenANT et al. 1989). Em A. catastomi Dana \& Herrick, 1837 variou de 500-600 
(WILSON 1902) e 1200 (DANA \& Herrick 1837 apud WILSON 1902). Em A. stizostehii Kellicott, 1880 variou de 50-300 (WILSON 1902). A. africanus depositou 421 ovos, em poucas horas (FRYER 1956).

Uma única fêmea de D. ranarum depositou 566 ovos, em cachos com 5-121 ovos, em um período de cinco dias (FRYER 1956). Neste trabalho com D. carvalhoi o número de ovos variou de 16 - 392 e, não foi possível separar, quais posturas eram de fêmeas adultas grandes e quais eram de fêmeas adultas pequenas. O número máximo de ovos de $D$. carvalhoi (392) está abaixo dos valores máximos encontrados para as fêmeas do gênero Argulus $(600,1200)$ e de D. ranarum (566).

Os ovos de A. americanus eram elipsóides e logo após a postura a cor era amarelo claro, depois escureciam. Os de A. catastomi eram elipsóides de coloração amarelo esbranquiçado, mudando para amarelo escuro e ocre. Os ovos de $A$. megalops também eram elípticos e amarelo-claro, escurecendo para castanho, mas não tão escuro quanto os de A. catastomi (WILSON 1902). Os de A. foliaceus eram elipsóides e brancos, tornando-se amarelo pálido. Os de A. stizostethi Kellicott, 1880 eram esféricos, inicialmente de cor branco leite tornando-se amarelo pálido. Os de A. africanus eram elipsóides (BOWER-SHORE 1940 apud AvENANT et al. 1989). Os de A. puthenveliensis eram ovais e de cor branca (THOMAS 1961).

Os ovos de D. ranarum eram elipsóides e logo após a postura eram de cor creme-amarela, tornando-se amarelo-escuro (FRYER 1959). Os ovos de D. carvalhoi também eram elipsóides. Assim com ao das outras espécies, após a postura a coloração dos ovos era amarela-esbranquiçada, posteriormente escureciam, ficando amarelo escuro e finalmente castanho. No caso do ovo tornar-se inviável a coloração tornava-se negra.

Nos ovos de A. africanus, a partir do décimo quarto dia, os contornos externos dos embriões e os olhos podiam ser vistos através da membrana, (FRYER 1956). Em A. puthenveliensis os olhos desenvolveram depois do sexto dia (Thomas 1961). Em D. carvalhoi os olhos foram observados a partir do nono dia da postura.

O desenvolvimento embrionário no ovo para quando a temperatura é inferior a $12^{\circ} \mathrm{C}$ e abaixo de $16^{\circ} \mathrm{C}$ a postura cessa. Os ovos de A. megalops necessitaram de 60 dias para eclodirem a $22^{\circ} \mathrm{C}$. Os de A. americanus de 18 dias a $18,5^{\circ} \mathrm{C}$. Os de A. catastomi 28-35 dias à $22^{\circ} \mathrm{C}$ (WILSON 1902). Os de A. africanus $30-36$ dias à $22-24^{\circ} \mathrm{C}$ (FRYER 1956). Os de A. japonicus 12 dias à $30^{\circ} \mathrm{C}$ e 60 dias à $15^{\circ} \mathrm{C}$ (TOKIOKA 1936). Em três posturas de $A$. puthenveliensis observadas a duração dos estágios embrionários, foi de 13 dias e 21 horas à $31^{\circ} \mathrm{C}$. O tempo de eclosão da massa de ovos foi de: $48 ; 54$ e 49 horas. A taxa de eclosão variou de 2,6-4,2 ovos por hora (THOMAS 1961). Os ovo de A. coregoni mantidos à $20^{\circ} \mathrm{C}$ eclodiram em 30 dias (SHIMURA 1981).

Os desenvolvimento embrionário dos ovos de $D$. ranarum levou de 27 a 35 dias à temperatura de $23,8^{\circ} \mathrm{C}$ (FRYER 1964). AVENANT et al. (1989) testou o desenvolvimento embrionário de $D$. ranarum à $24^{\circ} \mathrm{C}$ mas, houve um grande desenvolvimento de protozoários, que destruiu todos os ovos. Os oves mantidos à temperatura da sala cerca de $20^{\circ} \mathrm{C}$ levaram 57 dias para eclodir. Neste trabalho o desenvolvimento embrionário dos ovos de $D$. carvalhoi levou 16 dias para completar e eclodir, a uma temperatura de $28^{\circ} \mathrm{C}$. 
O desenvolvimento embrionário em Branchiura pode ser de dois tipos. Anapomórfico, as larvas nascem sem terminar o desenvolvimento embrionário, por isto passam menos tempo dentro do ovo, eclodem como náuplio e fixam-se ao hospedeiro por meio dos espinhos distais da maxílula. Depois da primeira muda todos os toracópodos são funcionais (MCLAUGHLIN 1980). As espécies com desenvolvimento anapomórfico são: A. americanus; A. catastomi; A. coregoni; A. foliaceus; A. japonicus; A. lepidostei Kellicott, 1878; A. maculosus Wilson, 1907 (ShIMURA 1981); A. tientsinensis Ku \& Wang, 1956; A. yui Wang, 1959; A. chinensis Ku \& Wang, 1955 (RUSTHON-MELLOR \& BOXSHALL 1994).

O segundo tipo é o epimórfico, as larvas completam todo o desenvolvimento embrionário, por isso passam mais tempo dentro do ovo. Eclodem como uma miniatura do adulto com todos os apêndices funcionais exceto as ventosas (MCLAUGHLIN 1980). As espécies que apresentam este tipo de desenvolvimento são: $A$. funduli Krøyer, 1863; A. megalops; A. puthenveliensis; A. stizostethi (SHIMURA 1981); A. africanus; A. siamensis Wilson, 1926 (RUSTHON-MELLOR \& BOXSHALL 1994) e D. ranarum (FRYER 1964; AvENANT et al. 1989). D. carvalhoi apresentou desenvolvimento epimórfico.

Dolops carvalhoi é um crustáceo parasita de peixes de água doce, com uma ampla distribuição na América do Sul. Tem ciclo de vida direto, baixa especificidade parasitária e parasitou em sete espécies de peixes no ambiente natural e, em sistemas artificiais, pode parasitar novos hospedeiros da fauna natural e espécies de peixes exóticas. Em qualquer trabalho que envolva introdução, reintrodução, transporte, repovoamento, cultivo de espécies naturais ou exóticas de peixes, muito cuidado deve se ter com espécies parasitas que apresentam estas características. Pois os danos podem ser catastróficos tanto para os estoques naturais quanto para as criações de peixes.

\section{REFERÊNCIAS BIBLIOGRÁFICAS}

Avenant, A.; J.G. van As \& G.C. Loots. 1989. On the hatching and morphology of Dolops ranarum larvae. (Crustacea: Branchiura). Jour. Zool. Lond. 217: 511-519.

Fryer, G. 1956. A report on the parasitic Copepoda and Branchiura of the fishes of lake Nyasa. Proc. Zool. Soc. Lond. 127 (3): 293-344.

1959. A report on the parasitic Copepoda and Branchiura of the fishes of lake Bangweulu.

Proc. Zool. Soc. Lond. 132 (4): 517-550.

. 1964. Further studies on the parasitic Crustacea of African freshwater fishes. Proc. Zool. Soc. Lond. 143: 79-102.

1968. The parasitc Crustacea of African freshwater fishes; their biology and distribution.

Jour. Zool. Lond. 156: 45-95.

KABATA, Z. 1970. Diseases of fishes, Crustacea as anemies of fishes. Gersey City, T.F.H., VII+171p. Lemos DE CASTRO, A. 1949. Contribuição ao conhecimento dos crustáceos argulídeos do Brasil (Branchiura: Argulidae), com descrição de uma nova espécie. Bol. Mus. Nac. Zool., n.s., Rio de Janeiro, 93: 1-8.

1986. Branchiura. Manual de identificação de invertebrados límnicos. Brasília, Ministério de Ciência e Tecnologia, 23p.

MaltA, J.C.O. 1982. Os argulídeos (Crustacea: Branchiura) da Amazônia brasileira, 2. Aspectos da ecologia de Dolops geayi (Bouvier, 1897) e Argulus juparanaensis Castro, 1950. Acta Amazonica, Manaus, 12 (4): 701-506. 
1984. Os peixes de um lago de várzea da Amazônia Central (lago Janauacá, rio Solimões) e suas relações com os crustáceos ectoparasitas (Branchiura: Argulidae). Acta Amazonica, Manaus, 14 (3/4): 355-372.

Malta, J.C.O. \& A.M.B. Varella. 1983. Os argulídeos (Crustacea: Branchiura) da Amazônia brasileira, 3. Aspectos da Ecologia de Dolops striata (Bouvier, 1899) e D. carvalhoi Lemos de Castro, 1949). Acta Amazonica, Manaus, 13 (2): 299-306.

McLaughlin, P.A. 1980. Comparative morphology of recent Crustacea. San Francisco, W.H. Freeman and Company, VIII+177p.

Ringuelet, R. 1943. Revisión de los Argulídos argentinos (Crustacea: Branchiura). Rev. Mus. La Plata 3 (19): 43-125.

Rushton-Mellor, S.K \& G.A. Boxshall. 1994. The developmental sequence of Argulus japonicus (Crustacea: Branchiura). Jour. Nat. His. 28: 763-785.

SHAFIR, A. \& J.G. VAN As. 1986. Laying, development and hatching of eggs of the fish ectoparasite Argulus japonicus (Crustacea: Branchiura). Proc. Zool. Soc. Lond. 210: 40-414.

SHImURA, S. 1981. The larval developmental of Argulus coregoni Thorell (Crustacea: Branchiura), Jour. Nat. Hist. 15: 331-348,

Токіока, T. 1936. Larval development and metamorphosis of Argulus japonicus. Mem. Coll. Sc. Kioto 12 (4): 93-114.

THomas, M.M. 1961. Observations on the habits and post-embryonic development of a parasitic Branchiura Argulus puthenveliensis Ramakrishna, 1958. Jour. Mar. Biol. Ass. India 3 (1/2): 75-86.

Weibezahn, F.H. \& T. Cobo. 1964. Seis argulideos (Crustacea: Branchiura) parasites de peces dulce acuicolas en Venezuela, com descricion de una nueva especie del genero Argulus. Acta Biologica, Caracas, 4 (2): 119-144.

WiLSON, C.B. 1902. North American parasite copepods of the family Argulidae, with bibliography of the group and a systematic review of all know species. Proc. U.S. Nat. Mus. 25: 625-742.

Recebido em 11.III.2002; aceito em 21.XI.2002. 